\title{
108. Adaptability evaluation of fully mechanized coal mining under the condition of typical coal seam
}

\author{
Xin-feng Wang ${ }^{1}$, Wen-zhi Ding ${ }^{2}$, Guan-shuang Tan ${ }^{3}$ \\ ${ }^{1}$ Hunan Key Laboratory for Geomechanics and Engineering Safety, Xiangtan University, \\ 411105 Xiangtan, China \\ ${ }^{1,2,3}$ School of Environment and Resources, Xiangtan University, 411105 Xiangtan, China \\ ${ }^{1}$ Corresponding author \\ E-mail: ${ }^{1}$ wangxinfeng110@126.com, ${ }^{2} 447162015 @ q q . c o m,{ }^{3} 1252787767 @ q q . c o m$
}

Received 27 October 2016; accepted 29 October 2016

DOI https://doi.org/10.21595/jme.2016.17888

\begin{abstract}
According to the characteristics of fully mechanized mining technique in thinner middle thick coal seam of Yuanzhuang coal mine, using the principle of fuzzy mathematics to build up the hierarchy analysis model of thinner middle thick coal seam mining technology of geological condition and comprehensive evaluation system. The influence factors and evaluation index of comprehensive evaluation model are obtained, the results can provide a theoretical basis and quantitative standard adaptability for thinner middle thick coal seam mining technology.
\end{abstract}

Keywords: the thinner middle thick coal seam, fully mechanized mining technique, the principle of fuzzy mathematics, comprehensive evaluation.

\section{Introduction}

At present our country mechanization technology for thin and thinner middle thick coal seam mining in thin is not mature, the coal seam mining is mostly used in blasting mining process. In the 1.3-2.0 $\mathrm{m}$ partial thin thick seam mining equipment manufacturing and difficult to use, the fully mechanized mining equipment especially thinner thick coal seam shearer technical performance is relatively backward, low production capacity and poor reliability. Especially when thinner thick seam is deep, complex geological conditions, mining such seams

of low economic efficiency, seriously restricting the development of thin, medium thick coal seam of coal mining technology [1-3]. Mass production practice shows that the economic benefits of mechanized mining, depends on the geological condition of coal seam. When the process system is determined, different coal seam condition will have different technical and economic effect. So, it is necessary to geological conditions of thinner middle thick coal seam fully mechanized coal mining geological condition evaluation.

\section{Project overview}

4111 working face of Yuanzhuang coal mine is the first mining face in the western mining area of Huaibei Mining Group. The working surface uses a single long wall mining method, fully caving method control the roof, the surface length is $131 \mathrm{~m}$, strike length is $623 \mathrm{~m}$, mining area is $81613 \mathrm{~m}^{2}$, mining reserves is $164600 \mathrm{t}$. The surface located nioumian syncline, the west wing, close to the axis, 4 coal seam, coal seam dip angle $10^{\circ}-18^{\circ}$, the average dip $12^{\circ}$; coal thickness of 1.3-2 m, the average coal thickness of $1.54 \mathrm{~m}$, which belongs to the partial thin to medium thick coal seam. Coal seam is basically stable, mining rates may be $100 \%$. The coal seam occurrence varies, generally contain a layer of gangue in coal seam, local two, the average thickness of $0.4 \mathrm{~m}$. Coal seam immediate roof is thick sandstone of $6 \mathrm{~m}$, immediate bottom is thick mudstone of $3.5 \mathrm{~m}$.

\section{Adaptability evaluation of fully mechanized mining technology}

\subsection{The purpose of evaluation}

The main purpose of the evaluation is to the future of coal mining geological conditions for 
coal classification, provide the basis for evaluation of adaptability of the mining process, the value of comprehensive evaluation is one of the main parameters to predict the level of working face per unit area yield, which provides the basis for the level of per unit area yield reliable predictions, thus reliable guarantee the sustained and stable development of the future rational mining configuration and mine. The main features are the evaluation of working face as basic assessment unit [4]. After the high concentration of mine production, particularly sensitive to changes in economic and technical index of the whole mine working face per unit area yield changes, in order to ensure sustained and stable high yield of high yield and high efficiency coal mine, must be detailed and comprehensive understanding of the future mining working face of geological conditions. This is not only conducive to the layout of roadway, but also conducive to the reasonable working face replacement [5].

\subsection{Evaluation method and content}

A lot of methods to evaluate the adaptability of fully mechanized working face coal seam geological conditions, such as comprehensive index method, TOPSIS method, RSR method, fuzzy comprehensive evaluation method, grey system method, this paper chooses a mature and strong method fuzzy comprehensive evaluation [6]. Fuzzy comprehensive evaluation method is a comprehensive evaluation method based on fuzzy mathematics, the method according to the membership degree theory to quality evaluation to quantitative evaluation, the clear results can solve the problem of quantifying fuzzy. According to 4111 working face of Yuanzhuang coal mine is thin and thick coal seam geological condition of technical and economic characteristics of the working face, coal seam geological conditions of fully mechanized mining process of fuzzy comprehensive evaluation.

The main contents of the fuzzy comprehensive evaluation method in 4111 working face of coal seam geological conditions for adaptability evaluation are as follows [7]:

(1) The geological conditions in Huaibei mining area, mining coal mining process, technical and economic effects and security of information and experience, analysis of the relationship between geological factors impact on the process and effect of mining;

(2) The geological factors of mining technology summary of the influence law as the basis, the application of fuzzy mathematics method, to determine the weight function and the evaluation index system of factors structure and geologic factors, geological factors subordinate factors, comprehensive evaluation model;

(3) According to geological data provide by Yuanzhuang coal mine, dividing the work surface to be on the block before mining, comprehensive evaluation model of working face block, get the evaluation value $P_{i j}^{\prime}\left(0 \leq P_{i j}^{\prime} \leq 1\right)$;

(4) On the basis of supplementary geological data and mining geological report disclosure,

the typical block (mined block) and evaluation, evaluation value after harvest $P_{i j}^{\prime \prime}$ $\left(0 \leq P_{i j}^{\prime} \leq 1\right), P_{i j}^{\prime \prime}$ as the evaluation more practical;

(5) Reliability analysis of pre-harvest evaluation, using $R_{i j}$ as the pre harvest evaluation reliability, $R_{i j}=P_{i j}^{\prime \prime} / P_{i j}^{\prime}$, because the excavation uncovered always encounter changes in geological conditions of exploration has not been able to discover, so the $R_{i j} \ll 1$;

(6) The working face block evaluation, to block before mining evaluation based on mining, evaluation of reliability as the correction coefficient, the block valuation value $P_{i j}=P_{i j}^{\prime} \times R_{i j}$.

\subsection{Structure factor evaluation and index}

According to the system, a simple and feasible principles, combined with Yuanzhuang coal mine 4111 working face coal seam characteristics, selection of geological structure, coal thickness, coal seam stability, dip angle of coal seam, the seam roof and floor, seam hard and block size of 7 combined factors comprehensively reflects the coal seam geological conditions, 
factor structure as shown in Fig. 1 evaluation of concrete [8].

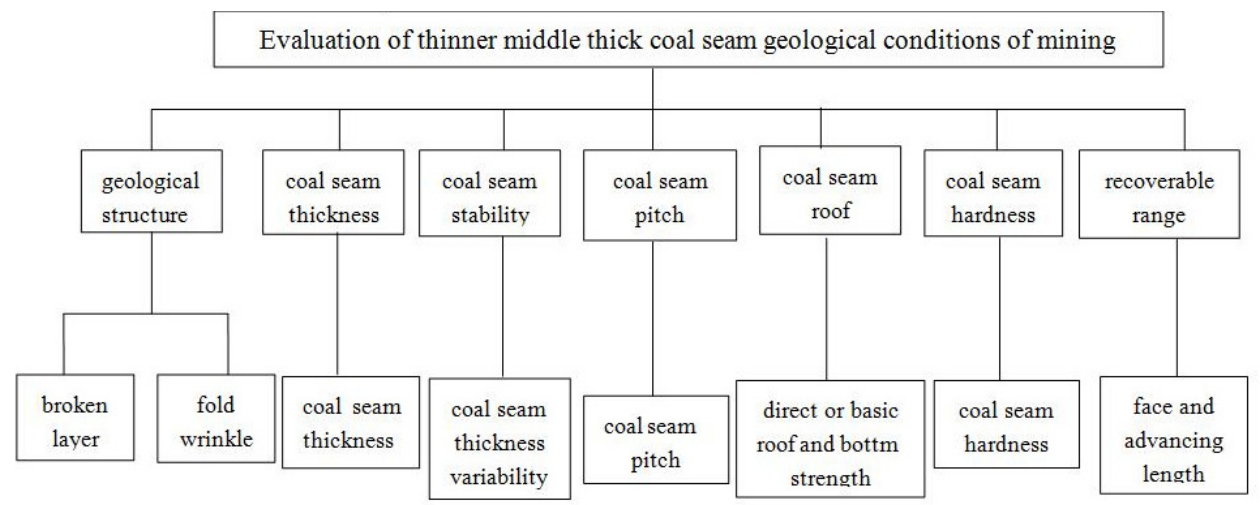

Fig. 1. The flow to implement virtual optimization design and analysis

\subsection{The membership function of evaluation factors}

The membership function is a mathematical tool used to characterize the fuzzy set. The fuzzy comprehensive evaluation of geological conditions of coal seam, quantitative description of fuzzy rule changes affecting the evaluation factors of membership function is the geological factors on mining effect. Construction of membership function can be integrated by data summary, literature survey, mathematical statistics and expert experience, the fuzzy linear inductive method, method of undetermined coefficients, multiple fuzzy statistics and other appropriate methods [9].

Because of the 4111 working face is too thin to medium thick coal seam, coal bed geological data, geological data of 4111 mining reveals is not comprehensive collection, so the construction of membership function of evaluation factors is difficult. But the partial thin medium thick coal seam in Huaibei mining area is rich in resources, mining of thin coal seam long-standing experience data, relative-abundance. The membership function of each evaluation in this paper is mainly based on the factors of geological exploration data, Yuanzhuang coal mine on the existing research results, combined with the China University of Mining and Technology professor Zhang Dongsheng about coal mining geological aspects of the process evaluation.

The membership function of each evaluation factors are as follows [8]:

(1) The influence of fault:

$\mu_{a}=\frac{2}{\left[1+\exp \left(0.0018+0.042 q_{1}+0.064 q_{2}+0.00071 q_{3}\right)\right]}$

(2) Fold effect:

$$
\mu_{b}=\frac{10}{\left[1+\exp \left(2.1975+0.0085 p_{1}+1.3028 p_{2}\right)\right]} \text {. }
$$

(3) Coal thickness variation index:

$$
\mu_{C^{\prime}}\left(C^{\prime}\right)=\left\{\begin{array}{l}
1-2\left(C^{\prime}\right)^{0.5}, \quad 0 \leq C^{\prime}<0.25, \\
0, \quad C^{\prime} \geq 0.25
\end{array}\right.
$$

(4) The thickness of coal seam: 
$\mu_{h}(h)= \begin{cases}0.25 h+0.05, & 1 \leq h<1.4, \\ 0.45 h-0.23, & 1.4 \leq h<2, \\ 0.12 h+0.43, & 2 \leq h<2.5, \\ 0.9 h-1.52, & 2.5 \leq h<2.8 \\ 1, & 2.8 \leq h<3.5, \\ -0.2 h+1.7, & 3.5 \leq h<4.5, \\ 0.7, & 4.5 \leq h<5, \\ 1, & h \geq 5 .\end{cases}$

(5) Coal seam pitch:

$\mu_{d}(d)= \begin{cases}1, & 0 \leq d<10 \\ 1.2-0.02 d, & 10 \leq d<20 \\ 1.275-0.025 d, & 20 \leq d<25 \\ 1.4-0.3 d, & 25 \leq d<30 \\ 1.7-0.04 d, & 30 \leq d<35 \\ 1-0.02 d, & 35 \leq d<45\end{cases}$

(6) Uniaxial compressive strength of immediate roof:

$\mu(r)= \begin{cases}0.4, & r<16 \\ \operatorname{Ln}\left(1.69 \times 10^{-4} \times r^{2}+0.012 r\right)+0.71, & 16 \leq r<30 \\ 1, & 30 \leq r<40 \\ 1.8-0.02 r, & 40 \leq h<80\end{cases}$

(7) The basic roof support:

$\mu_{N}(N)= \begin{cases}0.05, & N<0.1, \\ 0.4363 \operatorname{Ln}(N)+1.0045, & 0.1 \leq N<1, \\ 1.8-0.02 N, & N \geq 1 .\end{cases}$

(8) False roof thickness:

$\mu_{h 0}\left(h_{0}\right)= \begin{cases}1, & h_{0}<0.2 \\ -3 h_{0}+1.6, & 0.2 \leq h_{0}<0.5 \\ 0.1, & h_{0} \geq 0.5\end{cases}$

(9) The bottom strength:

$\mu_{Q}(Q)= \begin{cases}0.1, & Q<7.2, \\ 0.51 \ln Q-0.91, & 7.2 \leq \mathrm{Q}<42, \\ 1, & Q \geq 42 .\end{cases}$

(10) Coal seam hardness:

$\mu_{R}(R)= \begin{cases}0.1667 R, & 3 \leq R<6, \\ 1, & 6 \leq R<12, \\ -0.04167 R+1.5, & 12 \leq R<18, \\ -0.01389 R+1, & 18 \leq R<36, \\ -0.0286 R+1.528, & 36 \leq h<50 .\end{cases}$

(11) The length of working face: 
$\mu_{c}(c)= \begin{cases}0.005 c-0.1, & 40 \leq c<60 \\ 0.015 c-0.7, & 60 \leq c<80 \\ 0.125 c-0.5, & 80 \leq c<100, \\ 0.00067 c+0.482, & 100 \leq c<130 \\ 0.0085 c-0.275, & 130 \leq c<150, \\ 1, & c \geq 150 .\end{cases}$

(12) The advancing length of working face:

$\mu_{s}(s)= \begin{cases}0, & s \leq 100 \\ 0.5 \ln (s)-2.7, & 100 \leq s<1000 \\ 1, & s \geq 1000\end{cases}$

\subsection{Analysis of evaluation factors of weight}

The weight factors evaluation of geological condition of coal seam, its essence is the quantitative description of the geological factors on the working face mining process influence the relative importance. To determine the weights of evaluation factors to the geological condition of coal seam mining law as the basis, taking the geological condition and mining process of mutual adaptation as the main content, make full use of the statistical data, research results and the experience of experts. At present, the most commonly used method is analytic hierarchy process $[9,10]$. This method based on the expert knowledge of each other between the importance of each factor, which is clear concept, simple and strong pertinence, used to determine the weight of each specific working face of evaluation factors is feasible. Application of analytic hierarchy process method to obtain the weights of evaluation factors such as shown in Table 1.

Table 1. Evaluation factors weight table of the geological condition of coal seam

\begin{tabular}{|c|c|c|c|}
\hline $\begin{array}{c}\text { Name of middle layer } \\
\text { factors }\end{array}$ & $\begin{array}{c}\text { The middle layer of } \\
\text { weight factors }\end{array}$ & $\begin{array}{c}\text { Name of based } \\
\text { factors }\end{array}$ & $\begin{array}{c}\text { Fundamental factors of } \\
\text { weight }\end{array}$ \\
\hline \multirow{2}{*}{ Geological structure } & 0.3585 & Broken layer & 0.2861 \\
\cline { 3 - 4 } Coal seam thickness & 0.1028 & Fold wrinkle & 0.0724 \\
\hline Coal seam stability & 0.0955 & $\begin{array}{c}\text { Coal seam } \\
\text { thickness }\end{array}$ & 0.1028 \\
\hline Coal seam pitch & 0.0427 & Coal seam stability & 0.0955 \\
\hline \multirow{2}{*}{$\begin{array}{c}\text { Coal seam roof } \\
\text { and floor }\end{array}$} & \multirow{2}{*}{0.2210} & $\begin{array}{c}\text { Direct roof } \\
\text { strength }\end{array}$ & 0.0427 \\
\cline { 3 - 4 } & Basic roof strength & 0.0813 \\
\cline { 3 - 4 } & False roof effect & 0.0534 \\
\cline { 3 - 4 } & \multirow{2}{*}{$\begin{array}{c}\text { Basic bottom } \\
\text { strength }\end{array}$} & 0.0079 \\
\hline \multirow{2}{*}{ Coal seam hardness } & \multirow{2}{*}{$\begin{array}{c}\text { Coal seam } \\
\text { hardness }\end{array}$} & 0.0784 \\
\hline \multirow{2}{*}{$\begin{array}{c}\text { Recoverable range } \\
\text { of working face }\end{array}$} & 0.1143 & $\begin{array}{c}\text { Arranged face } \\
\text { length }\end{array}$ & 0.0652 \\
\cline { 3 - 4 } & & $\begin{array}{c}\text { Advancing face } \\
\text { length }\end{array}$ & 0.0468 \\
\hline Total & 1 & Total & 0.0675 \\
\hline
\end{tabular}

\subsection{Fuzzy comprehensive evaluation model}

The weight distribution of geological conditions of coal seams of each evaluation factor is determined, weighted sum method to construct the comprehensive evaluation model. In $U$ coal seam geological condition evaluation of sample set, $U=\left\{u_{i j}\right\}, i=1, \ldots, m, j=1, \ldots n, i$ is coal 
block number, $j$ is the serial number of evaluation factors, $n$ is the number of evaluation factors, $u_{i j}$ is $i$ coal block section $j$ factors with eigenvalues, the matrix can be expressed as:

$U=\left[\begin{array}{llll}u_{11} & u_{12} & \ldots & u_{1 n} \\ u_{21} & u_{22} & \ldots & u_{2 n} \\ \ldots & \ldots & \ldots & \ldots \\ u_{m 1} & u_{m 2} & \ldots & u_{m n}\end{array}\right]$.

The membership functions of the previous section $u_{i j}$ into the calculation, we can get the membership $w_{i j}$ of $u_{i j}$, so the index value matrix can be transformed into index membership degree matrix:

$W=\left[\begin{array}{cccc}w_{11} & w_{12} & \cdots & w_{1 n} \\ w_{21} & w_{22} & \cdots & w_{2 n} \\ \cdots & \cdots & \cdots & \cdots \\ w_{m 1} & w_{m 2} & \cdots & w_{m n}\end{array}\right]$

Let $T$ as the set of weights of evaluation factors, $T=\left(t_{1}, t_{2}, \ldots, t_{n}\right)$, we can get a comprehensive evaluation model of geological conditions of mining technology of working face by using the method of linear weighted average:

$P=\frac{1}{m} \sum_{i=1}^{m} T W^{T}$.

In this type: $P$ is the comprehensive evaluation value.

The comprehensive evaluation value is greater, the coal seam geological conditions of fully mechanized mining can obtain better technical and economic effect.

\subsection{Value and calculating the membership degree characteristics}

Establishing the evaluation system model will feature various geological factors value by the member ship function, and calculation of membership. Characteristics of various factors obtained geological data available from Yuanzhuang coal mine, respective into corresponding function, which can calculate the membership degree of each index. Characteristic indexes of 4111 working face value and membership function is shown in Table 2 .

Table 2. Evaluation indexes and membership list

\begin{tabular}{|c|c|c|c|c|}
\hline Name & \multicolumn{2}{|c|}{ Influencing factors } & Characteristic value & Membership \\
\hline \multirow{5}{*}{ Geological structure } & \multirow{3}{*}{ Broken layer } & Fault density & 22.6 & \multirow{3}{*}{0.52} \\
\hline & & Fault length & 0.8 & \\
\hline & & Fault throw & 12.7 & \\
\hline & \multirow{2}{*}{ Fold wrinkle } & Fold section coefficient & 0.0015 & \multirow{2}{*}{1.0} \\
\hline & & Fold surface coefficient & $6.3 \mathrm{e}-07$ & \\
\hline Coal seam thickness & \multicolumn{2}{|c|}{ Coal seam thickness $(\mathrm{m})$} & 1.54 & 0.6 \\
\hline Coal seam stability & \multicolumn{2}{|c|}{ Coal thickness variation coefficient } & 0.28 & 0.96 \\
\hline Coal seam pitch & \multicolumn{2}{|c|}{ The average coal seam inclination } & 14 & 1.0 \\
\hline \multirow{4}{*}{$\begin{array}{l}\text { Coal seam roof } \\
\text { and floor }\end{array}$} & \multicolumn{2}{|c|}{ Direct roof strength } & 22 & 0.8 \\
\hline & \multicolumn{2}{|c|}{ Basic roof strength } & 1.8 & 0.98 \\
\hline & \multicolumn{2}{|c|}{ False roof effect } & 0.5 & 1.0 \\
\hline & \multicolumn{2}{|c|}{ Basic bottom strength } & 22 & 0.73 \\
\hline Coal seam hardness & \multicolumn{2}{|c|}{ The comprehensive strength of coal seam } & 18 & 0.82 \\
\hline \multirow{2}{*}{$\begin{array}{l}\text { Recoverable range } \\
\text { of working face }\end{array}$} & \multicolumn{2}{|c|}{ Arranged face length } & 131 & 1.0 \\
\hline & \multicolumn{2}{|c|}{ Advancing face length } & 623 & 1.0 \\
\hline
\end{tabular}




\subsection{The results of comprehensive evaluation and classification}

After determining the index weight and the membership degree of evaluation, evaluation and calculation can be pre-harvest mechanized process adaptability according to the comprehensive evaluation model of the formula:

$P_{0}=\frac{1}{m} \sum_{i=1}^{m} T W^{T}\left[\begin{array}{l}0.2861 \\ 0.0724 \\ 0.1028 \\ 0.0955 \\ 0.0427 \\ 0.0813 \\ 0.0534 \\ 0.0079 \\ 0.0784 \\ 0.0652 \\ 0.0468 \\ 0.0675\end{array}\right]^{T}\left[\begin{array}{c}0.52 \\ 1.0 \\ 0.6 \\ 0.96 \\ 1.0 \\ 0.8 \\ 0.98 \\ 1.0 \\ 0.73 \\ 0.82 \\ 1.0 \\ 1.0\end{array}\right]=0.77$

According to the comprehensive evaluation of the geological condition of coal seam in Yuanzhuang coal mine, the value of the evaluation of the working surface can be divided into four levels, as is shown in Table 3. Through the calculation of comprehensive evaluation, the final face value is 0.68 , the comprehensive evaluation of 4111 working face, which belongs to the 2 level, mining condition is good, can be used in fully mechanized mining technique, the theory of fully mechanized mining technology in 4111 working face can achieve high yield and high efficiency.

Table 3. Classification analysis in fully mechanized face of Yuanzhuang coal mine

\begin{tabular}{|c|c|c|}
\hline Evaluation grade & Evaluation results & Mining conditions \\
\hline 1 Level & $>0.8$ & Much better \\
\hline 2 level & $0.65-0.80$ & Good \\
\hline 3 level & $0.50-0.65$ & General \\
\hline 4 level & $<0.5$ & Relatively poor \\
\hline
\end{tabular}

\section{Conclusions}

1) Based on the general situation of geological engineering mine, using the theory of fuzzy mathematics to set up a comprehensive evaluation of thick seam mining technology of thin geological condition system, on the face can carry out comprehensive quantitative analysis.

2) Through the fuzzy comprehensive evaluation model, analysis of the weight and membership degree of evaluation factors structure index and evaluation factors, the evaluation of the geological condition of coal seam in Yuanzhuang coal mine are obtained. In order to determine the comprehensive evaluation of the working surface belongs to 2 grade, mining condition is good, it can be used in fully mechanized mining technique, the theory of fully mechanized mining technology in the working face can achieve high yield and high efficiency.

\section{Acknowledgements}

This work is supported by Hunan Key Laboratory of Geomechanics and Engineering Safety (Grant No. 16GES10) and Xiangtan University Natural Science Foundation (Grant No. 15XZX41). 


\section{References}

[1] Liu C. Complicated Coal Seam Mining Technology and Equipment Selection of Evaluation. Auhui University of Science and Technology, Huainan, 2015.

[2] Wang X. J., Qian X. S., Ma L. Q. Research on large mining height technique for thick coal seams. Journal of Mining and Safety Engineering, Vol. 26, Issue 2, 2009, p. 212-216.

[3] Chen Y. H., Tang Z. L. Study on composite mechanized technique for medium-thickness coal sheet. HeBei Coal, Vol. 12, Issue 5, 2005, p. 55-56.

[4] Li Q., Chen Z. W., Yu W. Application of fully mechanized mining technique in thin coal seam under deep coal mine. Journal of Xi'an University of Science and Technology, Vol. 29, Issue 1, 2009, p. 31-35.

[5] Zhang L. J., Zhang L. Comprehensive assessment on suitability of fully mechanized coal mining technology in thin seam. Coal Science and Technology, Vol. 34, Issue 6, 2006, p. 43-45.

[6] Wu F. D., Hu N. L., Li S. G. Fuzzy evaluation of mining method for working face with large mining height in Dahaize coal mine. Safety in Coal Mines, Vol. 47, Issue 6, 2016, p. 206-208.

[7] Wang X. F., Gao M. Z. Comprehensive assessment on suitability of full mechanized coal mining technology in the thinner middle thick coal seam. Proceedings of International Mining Conference on Theory and Technique of Coal Mining and Disaster Prevention in Deep Mines, 2014, p. 161-166.

[8] Zhang D. S., Zhang X. C., Yan X. F. Fuzzy evaluation on geological conditions of coal seam in China. Proceedings of the 29th International Symposium on Computer Applications in the Mineral Industries. A. A. Balkema, Beijing, 2001, p. 25-27.

[9] Zhang D. S., Zhang J. X., Zhang X. C. Fuzzy evaluation on coal seam geological condition of coal face. Journal of Systems Engineering, Vol. 17, Issue 3, 2002, p. 252-256.

[10] Mao Z. H. Evaluation of geological condition mining technology in fully mechanized top-coal caving face. Ground Pressure and Strata Control, Vol. 19, Issue 4, 2002, p. 86-88. 